\title{
Electronic Transport Properties of Transition-Metal Terminated Trigonal Graphene Nanoribbons
}

\author{
Fanhua Meng, Minghua Zhang, Wen Liu* \\ Physics and Information Engineering Department, Jining University, Qufu, China \\ Email: *liuwenjnxy@163.com \\ Received 7 March 2016; accepted 25 July 2016; published 28 July 2016 \\ Copyright (C) 2016 by authors and Scientific Research Publishing Inc. \\ This work is licensed under the Creative Commons Attribution International License (CC BY). \\ http://creativecommons.org/licenses/by/4.0/

(c) (;) Open Access

\begin{abstract}
With the non-equilibrium Green's function method and density functional theory, we have studied the electronic properties of trigonal graphene nanoribbons, with Fe terminal and $\mathrm{H}$ terminal, coupled to gold electrodes. Rectification behavior can be observed when the electrode-molecule contact distance is larger than $2.2 \AA$. The electronic transport is greatly improved in case of Fe terminal which is analyzed in terms of transmission spectra and density of states.
\end{abstract}

\section{Keywords}

Trigonal Graphene Nanoribbons, Electronic Transport, Fe Terminal, Transmission Spectra

\section{Introduction}

Carbon-based nanostructures have emerged as one of the most promising candidates for nanoelectronics in recent years. Much attention has been paid to developing graphene electronics. Due to their interesting electronic properties, such as the giant carrier mobility at room temperature [1], the anomalous quantum hall effect [2], and quantum blockade [3], narrow graphene nanoribbons (GNRs) has a promising prospect of application in graphene-based devices.

With the development of experimental technology, it has become possible to tailor a GNR into well-defined shapes by cutting a grapheme along specific single crystallographic directions [4]. By tailoring GNRs into a triangular shape, Chen et al. have designed a rectifying and perfect spin filtering electronic device based on AGNRs [5]. Deng et al. investigated the effects of side groups on the electronic transport properties of the trigonal grapheme flake and found that the rectifying rations and direction could be significantly tuned by the type and the attached positions of side groups [6]. A similar triangular GNR nanostructure has also been investigated

\footnotetext{
*Corresponding author.
}

How to cite this paper: Meng, F.H., Zhang, M.H. and Liu, W. (2016) Electronic Transport Properties of Transition-Metal Terminated Trigonal Graphene Nanoribbons. Journal of Modern Physics, 7, 1359-1363. 
in which the vertex carbon of the GNR is substituted by B and N. The rectification behavior was observed because a potential barrier similar to the p-n junction was formed in the B-N region of the central molecule [7].

The presence of metal atoms adsorbed on GNRs changes its electronic and magnetic properties and thus is of great interest in physics. Wang et al. investigated the structure, electronic and magnetic properties of metal-terminated zigzag GNR (ZGNR) [8]. Cao et al. investigated the magnetic line defects located at the ribbon edge and spin-filter effect was observed [9].

In this paper, we aim to construct a two-probe nanodevice based on Fe-terminated triangular ZGNR and compare the transport properties of Fe-terminated triangular ZGNR system and H-terminated triangular ZGNR system. As it turns out, it is interesting to find that in the presence of Fe-terminal, the electronic transport is greatly enhanced compared with H-terminal.

\section{Model and Method}

Our modeling setup is depicted in Figure 1. Fe-terminated and H-terminated triangular ZGNR system are denoted as Z-H (Figure 1(a)) and Z-Fe (Figure 1(b)), respectively. The vacuum layers between two adjacent images along $\mathrm{x}$ and $\mathrm{y}$ directions are set to $10 \AA$. The molecular device we studied is divided into three regions: left electrode, right electrode and scattering region. The scattering region involves central molecule and the first three gold layers near it.

The geometry of these two systems is first optimized with a residual force of $0.05 \mathrm{eV} / \AA \AA$. After the structure relaxation, we calculate the electronic transport properties of the system by the well test ATK package [10], which adopts a non-equilibrium Green function (NEGF) method combined with density functional theory (DFT). The single-zeta polarization (DZP) basis set for valence electrons is adapted. The Perdew-Burke-Ernzerhof (PBE) formulation of the generalized gradient approximation (GGA) exchange correlation function is used. Core electrons are modeled with Troullier-Martins nonlocal pseudopotential. An energy cut off of 200 Ry is used to define the real-space grid for numerical calculations in the electron density. The electronic Brillouin zone integration is sampled with a $1 \times 1 \times 100$ uniform k-point mesh. The current is calculated using the widely accepted Landauer-Büttiker formula [7]:

$$
I=\frac{2 e}{h} \int T\left(E, V_{b}\right)\left[f_{L}\left(E-\mu_{L}\right)-f_{R}\left(E-\mu_{R}\right)\right] \mathrm{d} E
$$

where $T\left(E, V_{b}\right)$ is the transmission coefficient for electrons with energy $E$ at bias voltage $V_{b}, f_{\mathrm{L} / \mathrm{R}}$ the Fermi function, and $\mu_{\mathrm{L} / \mathrm{R}}$ the electrochemical potential of the left/right electrode.

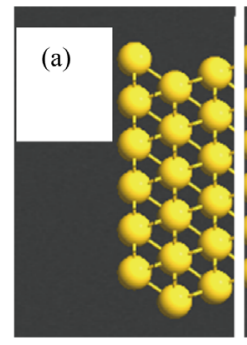

the left region

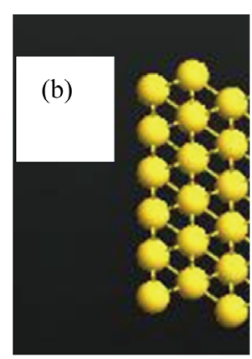

the left region

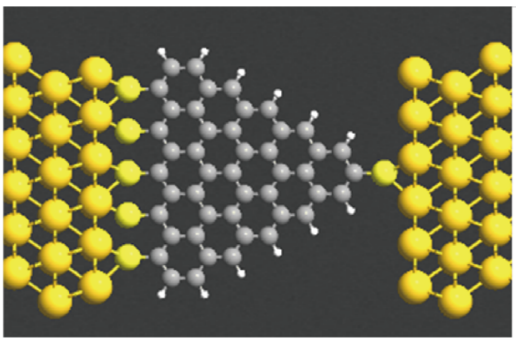

the center region

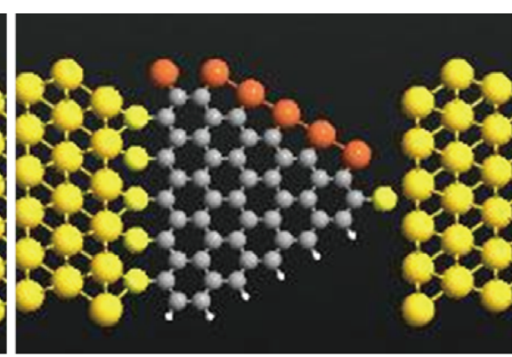

the center region

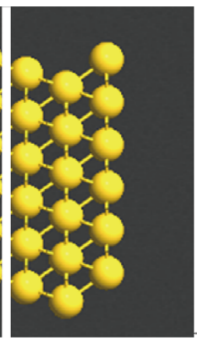

the right region

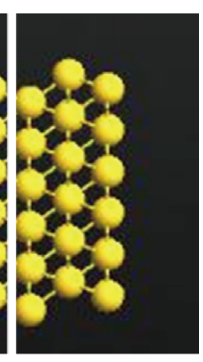

the right region

Figure 1. The geometric structures (a) Z-H and (b) Z-Fe. 


\section{Results and Discussion}

First, we calculate the self-consistent current-voltage (I-V) characteristic for Z-H system in a bias range from $-2.0 \mathrm{~V}$ to $2.0 \mathrm{~V}$ which is shown in Figure 2(a). We have discussed the effect of the electrode-molecule contact distance on the electronic transport and found that when the left and right contact distance varies from $1.8 \AA$ to $2.2 \AA$, no obvious rectifying behavior is observed although the central molecule itself has asymmetric structure. Four I-V curves corresponding to different contact distances are taken for example, among which three ones are obtained with electrode-molecule contact distance less than $2.3 \AA$ and the fourth with the right contact distance equal to $2.3 \AA$. In the whole, the I-V curves are asymmetric due to the asymmetric molecule structure and they have similar features in the bias range from $-1.0 \mathrm{~V}$ to $1.0 \mathrm{~V}$. In such a bias range, there is no obvious current through the GNR. The current shows remarkable enhancement until the bias increases to $1.0 \mathrm{~V}(-1.0 \mathrm{~V})$. The situation in negative bias is similar so that no rectification is observed. However, when the right and left contact distances are changed to $2.3 \AA$ and 1.8 Á respectively, obvious rectifying behavior is observed. This is reasonable because the strength asymmetry of the right and left electrode-molecule coupling could lead to current rectification.

Next we turn to the Fe-terminated system. In order to illustrate the effect of Fe-terminal on the electronic transport, we plot in Figure 2(b) the I-V curves for Z-H and Z-Fe for comparison. The I-V curves are obtained numerically in the bias range $(-2.0,2.0) \mathrm{V}$ with an interval of $0.2 \mathrm{~V}$. The I-V curves of Z-Fe are also calculated with the contact distance varying from $1.8 \AA$ to $2.2 \AA$ and one I-V curve is chosen as an example. It is found that the electronic transport is greatly enhanced in Z-Fe compared with Z-H. The current of Z-Fe increases by one order of magnitude.

Transmission spectra analysis provides us a most intuitive picture for understanding the I-V characteristic. We have calculated transmission spectra in the bias range $(-2.0,2.0) \mathrm{V}$ with an interval of $0.2 \mathrm{~V}$. Without loss of generality, we plot in Figure 3 the transmission spectra for Z-H and Z-Fe at bias $0,1.0 \mathrm{~V}$ and $2.0 \mathrm{~V}$ as representative ones. As the current is obtained from the Landauer-Büttiker formula mentioned above, the magnitude of current in fact depends on the transmission coefficient in the integral regions, namely, the size of the integral area in the bias window. The dashed lines denote the corresponding bias windows at different biases. As can be seen from Figure 3(a), Figure 3(c) and Figure 3(e), no transmission peaks appear around the Fermi level at $V_{b}$ $=0$. In case of $V_{b}=1.0 \mathrm{~V}$ one peak appears and enter the bias window. Therefore, there is relatively weak enhancement in current. When the bias is further increased to $2.0 \mathrm{~V}$, the peak inside the window splits into two ones and the current is larger than that at $V_{b}=1.0 \mathrm{~V}$. The change of transmission spectra with the increase of bias is different for Z-Fe. From Figure 3(b), Figure 3(d) and Figure 3(f) it can be found that much more peaks appear no matter at lower bias or at higher bias. As the bias increases more and more transmission peaks enter the bias window, which leads to continuous enhancement in current as shown in Figure 2(b). As a result, the current of Z-Fe is much larger than that of Z-H.

To further rationalize the significant difference in the electronic transport of these two kinds of systems, we plot in Figure 4 the density of states (DOS) at $V_{b}=0,-1.0 \mathrm{~V}, 1.0 \mathrm{~V}$. It is clear that there are more electronic states around the Fermi level in Z-Fe than that in Z-H, which undoubtedly contributes to the stronger transport
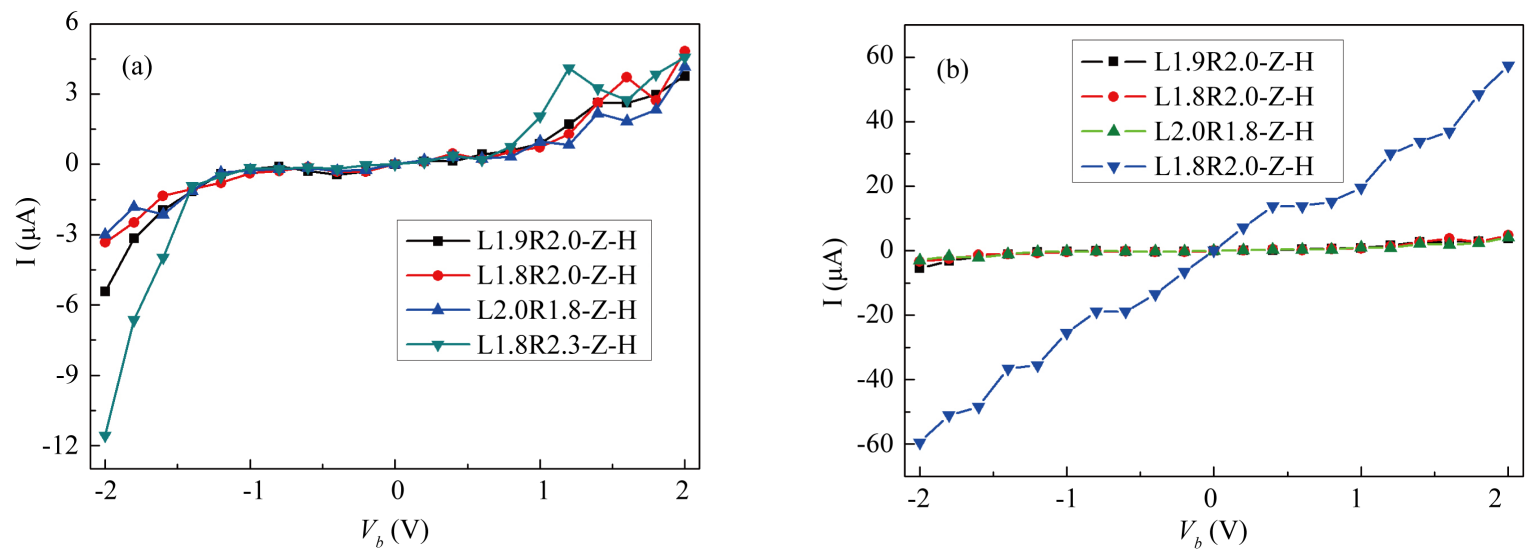

Figure 2. Calculated $I-V$ curves for Z-H and Z-Fe systems. 

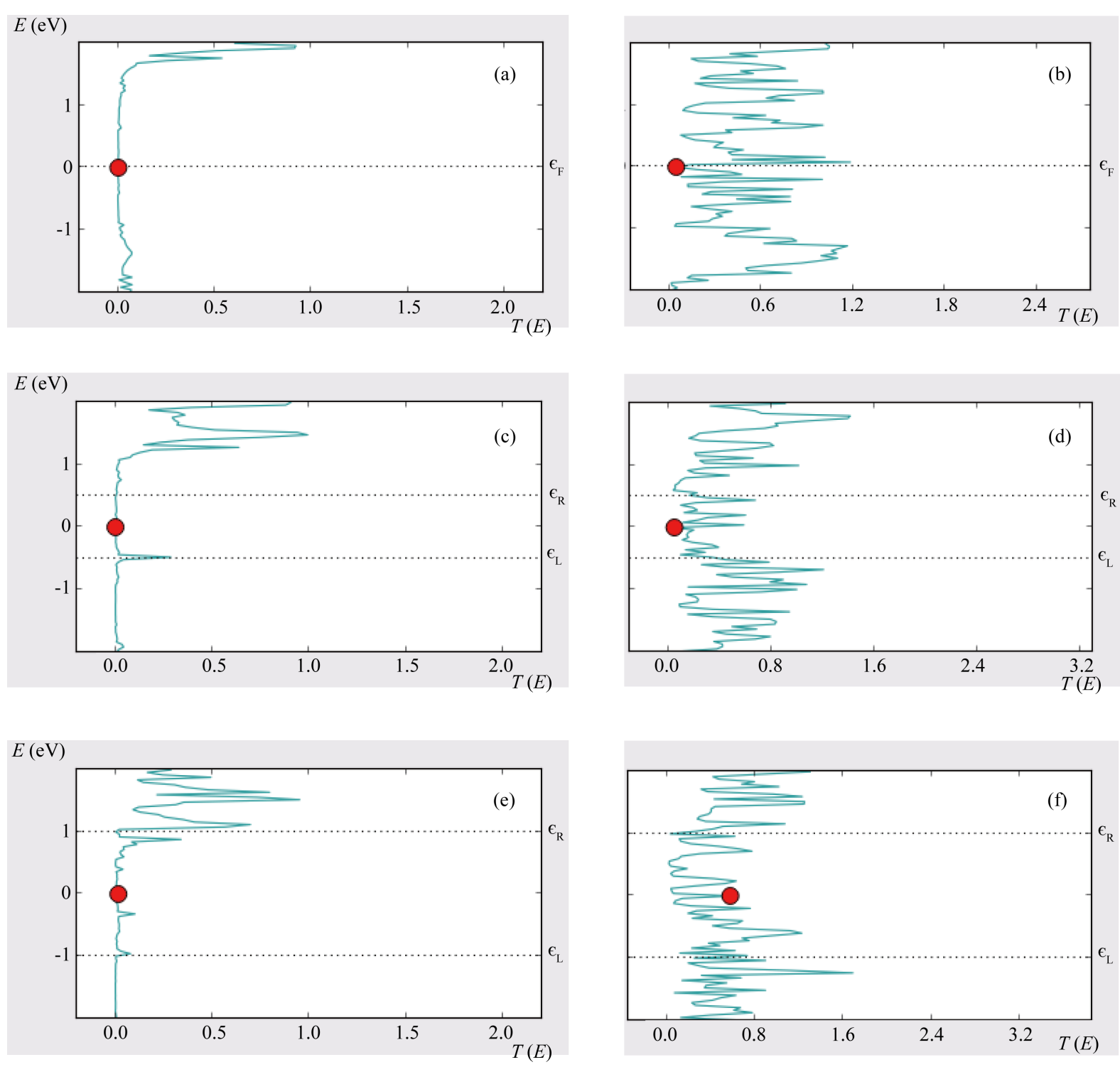

Figure 3. Transmission spectra for Z-H (the left panel) and Z-Fe (the right panel) at different bias voltages $V_{b}=0,1.0 \mathrm{~V}$, and $2.0 \mathrm{~V}$. (a) $V_{b}=0$; (b) $V_{b}=0$; (c) $V_{b}=1.0 \mathrm{~V}$; (d) $V_{b}=1.0 \mathrm{~V}$; (e) $V_{b}=2.0 \mathrm{~V}$; (f) $V_{b}=2.0 \mathrm{~V}$.
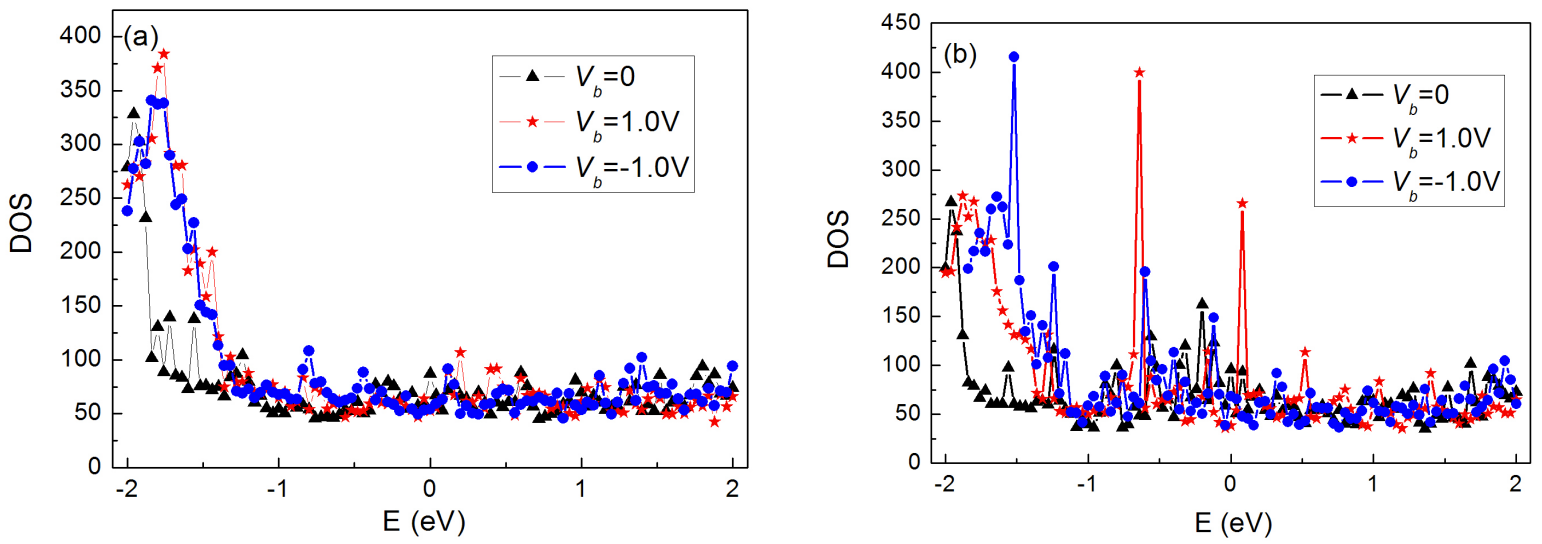

Figure 4. Density of state for (a) Z-H and (b) Z-Fe at different bias voltages $0,-1.0 \mathrm{~V}, 1.0 \mathrm{~V}$. 
ability of Z-Fe.

\section{Summary}

We have constructed two-probe nanodevices based on triangular ZGNR. Effect of electrode-molecule contact distance on the electronic transport is discussed and it is found that when the left and right contact distance varies from $1.8 \AA$ to $2.2 \AA$, no obvious rectifying behavior is observed although the central molecule itself has asymmetric structure. The transport properties of Fe-terminated and H-terminated triangular ZGNRs are compared. As it turns out, it is interesting to find that in the presence of Fe-terminal, the electronic transport is greatly enhanced compared with $\mathrm{H}$-terminal. The significant difference in the electronic transport of these two kinds of systems is attributed to the much more transmission peaks around the Fermi level in Fe-terminated system.

\section{Acknowledgements}

Liu Wen thanks the support Shandong Province Higher Educational Science and Technology Program (Grant No. J13LJ53) and the Natural Science Foundation of Shandong Province (No. ZR2014AL013).

\section{References}

[1] Morozov, S.V., Novoselov, K.S. and Katsnelson, M.I. (2008) Physical Review Letters, 100, Article ID: 016602.

[2] Geim, A.K. and Novoselov, K.S. (2007) Nature Materials, 6, 183-191. http://dx.doi.org/10.1038/nmat1849

[3] Zhang, Y.Y., Hu, J.P., Bernevig, B.A., Wang, X.R., et al. (2008) Physical Review B, 78, Article ID: 155413.

[4] Campos, L.C., Manfrinato, V.R., Yamagishi, J.D.S., et al. (2009) Nano Letters, 9, 2600-2603. http://dx.doi.org/10.1021/nl900811r

[5] He, J. and Chen, K.Q. (2012) Journal of Applied Physics, 112, Article ID: 114319.

[6] Deng, X.Q., Tang, G.P. and Guo, C. (2012) Physics Letters A, 376, 1839-1844. http://dx.doi.org/10.1016/j.physleta.2012.04.021

[7] Deng, X.Q., Zhang, Z.H. and Tang, G.P. (2012) Applied Physics Letters, 100, Article ID: 063107.

[8] Wang, Y. and Cheng, H.P. (2011) Physical Review B, 83, Article ID: 113402.

[9] Cao, C., Chen, L.N., Long, M.Q., Huang, W.R. and Xu, H. (2012) Journal of Applied Physics, 111, Article ID: 113708.

[10] Brandbyge, M., Mozos, J.L., Ordejón, P., Taylor, J. and Stokbro, K. (2002) Physical Review B, 65, Article ID: 165401.

\section{Submit or recommend next manuscript to SCIRP and we will provide best service for you:}

Accepting pre-submission inquiries through Email, Facebook, LinkedIn, Twitter, etc.

A wide selection of journals (inclusive of 9 subjects, more than 200 journals)

Providing 24-hour high-quality service

User-friendly online submission system

Fair and swift peer-review system

Efficient typesetting and proofreading procedure

Display of the result of downloads and visits, as well as the number of cited articles

Maximum dissemination of your research work

Submit your manuscript at: http://papersubmission.scirp.org/ 